\title{
Fever and Serology
}

\author{
Malavalli V Bhavana
}

\section{Abstract}

Fever is a common symptom encountered in the clinical practice. This can provide vital clues about the underlying condition. This is a brief report on the role of serological investigations in a patient with fever.

Keywords: Fever, Infections, Serology.

Pediatric Infectious Disease (2020): 10.5005/jp-journals-10081-1245

\section{INTRODUCTION}

Fever can be referred to a Pandora's box, as it is surrounded by so many mysteries. The proper investigation of fever is important, as it provides important diagnostic clues. This review discusses the microbiological experiences with fever from the perception of a physician, which could assist in fever investigation.

\section{Epidemiology and Causes}

Fever is highly variable. In Western countries, inflammatory causes are more common and infection plays a major role in causing fever in low- and middle-income countries. The most commonly reported infections are enteric fever, brucellosis, tuberculosis, endocarditis, and intra-abdominal abscesses. ${ }^{1}$

The infectious etiologies of fever include enteric fever, tuberculosis, dengue/viral fever, malaria, leptospirosis, brucellosis, typhus fever, focal abscesses, infectious mononucleosis, and infective endocarditis. The noninfectious etiologies include connective tissue disorders, autoimmune disorders, and malignancies.

\section{History Collection}

The methodology to investigate a fever starts from the history. A careful history should be comprised of previous infectious illnesses, family history of infection, exposure to similar infections, residence and country of origin, recent travel, zoonotic exposure, and leisure activities. Physical examination requires special attention to the eyes, heart, spinal tenderness point, liver/spleen, lymphadenopathy, skin lesions, and oropharynx. ${ }^{1}$

\section{Basic Laboratory and Radiological INVESTIGATIONS}

The basic laboratory investigations include complete blood count (CBC), peripheral blood smear, erythrocyte sedimentation rate, C-reactive protein (CRP), liver function tests, and blood and urine culture. Radiological investigations such as the chest radiograph, CT, and MRI scans of the abdomen and pelvis are performed, if required. ${ }^{1}$

The $C B C$ is the most important nondefinite investigation performed in cases with fever. The observations from $C B C$ are suggestive of the following diseases:

- Leukopenia: Enteric fever, TB, HIV, SLE

- Leukocytosis: Pyogenic infection, vasculitis

- Reactive lymphocytosis: EBV, CMV
Department of Microbiology, Manipal Hospitals, Bengaluru, Karnataka, India

Corresponding Author: Malavalli V Bhavana, Department of Microbiology, Manipal Hospitals, Bengaluru, Karnataka, India, Phone: +91 9986157911, e-mail: bhavana224@gmail.com

How to cite this article: Bhavana MV. Fever and Serology. Pediatr Inf Dis 2020;2(1):29-31.

Source of support: Nil

Conflict of interest: None

- Eosinophilia: Drug reactions, parasitic infections

- Eosinopenia: Enteric fever

- Thrombocytosis: Pyogenic infection, inflammation

- Thrombocytopenia: Malaria, SLE, HIV

- Pancytopenia: Bone marrow infiltration, SLE

The liver function test also plays a vital role in the investigation. Elevated alanine transaminase (ALT)/aspartate transaminase (AST) indicates viral hepatitis or infectious mononucleosis. Elevated alkaline phosphatase and gamma glutamyl transpeptidase indicate granulomatous hepatitis, sepsis, or cholangitis. ${ }^{2}$

Segal et al. have reported CRP as the most useful marker in children to differentiate bacterial and viral infections. The CRP increases during the first 36 hours of fever and declines more rapidly with viral infections. In a patient with fever duration $>24$ hours, if CRP is $>11 \mathrm{mg} / \mathrm{dL}$, the probability of having a bacterial infection is $75 \%$; whereas if CRP is $\leq 5 \mathrm{mg} / \mathrm{dL}$, the chance of a bacterial infection is unlikely ( $>95 \%$ accuracy). ${ }^{3}$

Procalcitonin is more specifically elevated in bacterial infections and the levels can correlate with the severity of sepsis. It can be used as a trend to monitor a sepsis patient, rather than a single diagnostic marker. ${ }^{4}$

\section{Enteric Fever}

Timing of tests is very important in the investigation of enteric fever. Week 1: Isolation of Salmonella from blood/bone marrow. Automated culture systems do better. The success of a culture lies in the blood volume and proper timing of the collection of the sample.

Week 2: The Widal by tube method is preferred. The slide method can also be used, but there are chances of false-positive results.

Week 3: Stool culture.

Week 4: Urine culture.

(0) The Author(s). 2020 Open Access This article is distributed under the terms of the Creative Commons Attribution 4.0 International License (https://creativecommons. org/licenses/by-nc/4.0/), which permits unrestricted use, distribution, and non-commercial reproduction in any medium, provided you give appropriate credit to the original author(s) and the source, provide a link to the Creative Commons license, and indicate if changes were made. The Creative Commons Public Domain Dedication waiver (http://creativecommons.org/publicdomain/zero/1.0/) applies to the data made available in this article, unless otherwise stated. 
As per the $\mathrm{WHO}$, there is no definitive role for rapid typhoid antibody tests like tubex, typhidot, typhidot rapid, and lgM dipstick, and there are no other molecular tests in the market for routine diagnosis. Culturing remains the gold standard. ${ }^{5}$

\section{Brucellosis}

IgM raises first and peaks at around 3 months of the infection. IgM ELISA is usually preferred, followed by the Brucella agglutination test. However, blood culture remains the gold standard, but prolonged incubation is required. ${ }^{6}$

\section{Dengue}

The NS1 antigen level elevates during the first 5 days of fever, followed by lgM and IgG in a primary infection. Secondary infection witnesses an accelerated IgG response. The serological markers include ELISA for NS1, IgM, and lgG. ${ }^{7}$ Real-time PCR is helpful during an acute viremic phase. The rapid test does not have any role.

\section{Chikungunya}

Markers include lgM, IgG, and circulating viruses. According to the testing algorithm by CDC, if the disease onset is $<6$ days, PCR should be performed and if it is $>6$ days, IgM ELISA should be carried out. ${ }^{8}$

\section{Leptospirosis}

Around $90 \%$ of the infection is presented as undifferentiated febrile illness. It is often misdiagnosed at onset as aseptic meningitis, influenza, hepatic disease, or fever (pyrexia) of unknown origin. The tests available for the diagnosis of leptospirosis include dark field microscopy, IgM ELISA, microscopic agglutination test, and PCR. Dark field microscopy requires 104 leptospires $/ \mathrm{mL}$ to be visible under the microscope. However, it lacks sensitivity and specificity. IgM ELISA has chances of false positivity. The gold standard is the microscopic agglutination test, followed by PCR. ${ }^{9}$

\section{Infectious Mononucleosis}

The immune response of infectious mononucleosis involves several antigens and antibodies, and they include early antigens, followed by heterophile antibodies and later viral capsid antigens (VCA IgM, VCA IgG, and Epstein-Barr nuclear antigen (EBNA)-1 lgG). A positive VCA IgM and negative VCA IgG and EBNA-1 IgG and a positive VCA $\lg M$ and $\lg G$ and negative EBNA-1 IgG indicate acute infection. A positive EBNA-1 IgG and VCA IgG indicate past infection. A late primary infection is indicated when all three markers are positive. ${ }^{10}$

\section{Malaria}

The target antigens are $P$. falciparum-specific proteins like histidinerich protein II or lactate dehydrogenase and pan-specific antigens (aldolase or pan-malaria pLDH). The PCR, generally used to confirm malaria infection, detects only $1-5$ parasites $/ \mu \mathrm{L}$ of blood. However, peripheral smear and rapid tests can detect 50-100 parasites/ $\mu \mathrm{L}$ of blood. ${ }^{11}$ Recently the Ministry of Health and Family Welfare prohibited the use of antibody-detecting rapid diagnostic tests for the diagnosis of malaria. ${ }^{12}$

\section{Typhus Fever}

Scrub typhus and Indian tick typhus predominate in India. The Weil-Felix test can be used only after 1 week and has low sensitivity and specificity due to cross-reactivity of Proteus antigens used. However, ELISA has good sensitivity and specificity. IgM ELISA and the $R$. conorii ELISA IgG/lgM kit are used correspondingly for the detection of scrub typhus and Indian tick typhus. ${ }^{13}$ Scrub typhus
IgM should be interpreted with caution, as IgM levels persist for a longer duration and there are possibilities for false-positive results when single serum samples are interpreted. ${ }^{11}$ Therefore, further research should focus on antigen detection assays. ${ }^{14}$

\section{Tuberculosis}

The Ministry of Health and Family Welfare, Government of India, has banned the use of inaccurate serological blood tests for the diagnosis of TB..$^{15}$ The Quantiferon TB gold test (QFT) measures the release of interferon gamma produced in whole blood in response to stimulation by the purified protein derivative. The Centers for Disease Control and Prevention (CDC) recommends initial and serial testing of persons with an increased risk for latent TB (recent immigrants, injection drug users, residents, and employees of prisons and jails) and also for individuals who are, by history, at low risk for latent TB but whose future activity might place them at increased risk for exposure (healthcare workers and military personnel). ${ }^{16}$ However, Quantiferon gold is contraindicated in the evaluation of suspected active tuberculosis; assessment of contacts of persons with infectious tuberculosis; screening of children aged $<17$ years, pregnant women, or for persons with clinical conditions that increase the risk for progression of latent to active TB; detection of latent TB after suspected exposure; confirmation of tuberculin skin test results; and diagnosis of M. avium complex disease. ${ }^{17}$

\section{Invasive Fungal Infections}

Biologic markers include galactomannan Aspergillus antigen and the fungal wall component (1-3)- $\beta-D$-glucan. However, it is associated with a high negative predictive value. The 2016 Infectious Diseases Society of America (IDSA) guidelines for aspergillosis recommends that galactomannan and $1,3-\beta-D$-glucan assays are useful in high-risk patients and are not recommended for routine blood screening in patients receiving antifungal therapy or prophylaxis, but can be applied to bronchoscopy specimens from these patients. ${ }^{18}$ Invasive fungal infection should be tested in conjunction with other methods for the diagnosis of invasive fungal infections and should precede antifungal therapy. Positive test results should be confirmed with a second new specimen or repeated from the initial specimen. ${ }^{19}$

\section{References}

1. Fever and Fever of Unknown Origin (FUO) [Internet]. Infectious Disease Advisor. 2016 [cited 2019 Jan 3]. Available from: https://www. infectiousdiseaseadvisor.com/infectious-diseases/fever-and-feverof-unknown-origin-fuo/article/609714/.

2. Minemura $M$, Tajiri K, Shimizu Y. Liver involvement in systemic infection. World J Hepatol 2014;6(9):632-642. DOI: 10.4254/wjh. v6.i9.632.

3. Segal I, Ehrlichman M, Urbach J, et al. Use of time from fever onset improves the diagnostic accuracy of C-reactive protein in identifying bacterial infections. Arch Dis Child 2014;99(11):974-978. DOI: 10.1136/ archdischild-2013-305640.

4. Lee $\mathrm{H}$. Procalcitonin as a biomarker of infectious diseases. Korean $J$ Intern Med 2013;28(3):285-291. DOI: 10.3904/kjim.2013.28.3.285.

5. WHO| Sensitivity and specificity of typhoid fever rapid antibody tests for laboratory diagnosis at two sub-Saharan African sites [Internet]. WHO. [cited 2019 Jan 3]. Available from: https://www.who.int/ bulletin/volumes/89/9/11-087627/en/.

6. Nielsen K, Yu WL. Serological diagnosis of brucellosis. Prilozi 2010;31(1):65-89.

7. Jyothi $P$, Metri BC. Correlation of serological markers and platelet count in the diagnosis of dengue virus infection. Adv Biomed Res 
[Internet] 2015;4 10.4103/2277-9175.150396Available from: https:// www.ncbi.nlm.nih.gov/pmc/articles/PMC4333486/.

8. Johnson BW, Russell BJ, Goodman CH. Laboratory diagnosis of chikungunya virus infections and commercial sources for diagnostic assays. J Infect Dis 2016;214(Suppl 5):S471-S474. DOI: 10.1093/infdis/ jiw274.

9. Budihal SV, Perwez K. Leptospirosis diagnosis: competency of various laboratory tests. J Clin Diagn Res 2014;8(1):199-202. DOI: 10.7860/ JCDR/2014/6593.3950.

10. De Paschale M, Clerici P. Serological diagnosis of Epstein-Barr virus infection: problems and solutions. World J Virol 2012;1(1):31-43. DOI: 10.5501/wjv.v1.i1.31.

11. Kalal BS, Puranik P, Nagaraj S, et al. Scrub typhus and spotted fever among hospitalised children in South India: clinical profile and serological epidemiology. Indian J Med Microbiol 2016;34(3):293. DOI: $10.4103 / 0255-0857.188315$.

12. Tangpukdee N, Duangdee $C$, Wilairatana $P$, et al. Malaria diagnosis: a brief review. Korean J Parasitol 2009;47(2):93-102. DOI: 10.3347/ kjp.2009.47.2.93.

13. Ministry of Health Prohibits Antibody Detecting Rapid Diagnostic Tests For Routine Diagnosis Of Malaria [Internet]. vLex. [cited 2019 Jan 3]. Available from: https://vlex.in/vid/ministry-of-healthprohibits-719407617.
14. Varghese GM, Rajagopal VM, Trowbridge P, et al. Kinetics of IgM and IgG antibodies after scrub typhus infection and the clinical implications. Int J Infect Dis 2018;71:53-55. DOI: 10.1016/ j.ijid.2018.03.018.

15. Saraswati K, Day NPJ, Mukaka M, et al. Scrub typhus pointof-care testing: a systematic review and meta-analysis. PLoS Negl Trop Dis 2018;12(3):e0006330. DOI: 10.1371/journal.pntd. 0006330.

16. Summary $\rightarrow$ Tuberculosis Blood Test Important Diagnosis And [Internet]. [cited 2019 Jan 3]. Available from: http://yousense. info/747562657263756c6f736973/tuberculosis-blood-testimportant-diagnosis-and.html.

17. Guidelines for Using the QuantiFERON [Internet]. [cited 2019 Jan 3]. Available from: https://www.cdc.gov/MMWR/preview/MMWRhtml/ rr5202a2.htm.

18. Patterson TF, Thompson GR, Denning DW, et al. Practice guidelines for the diagnosis and management of aspergillosis: 2016 update by the infectious diseases society of America. Clin Infect Dis 2016;63(4): e1-e60. DOI: 10.1093/cid/ciw326.

19. Wright WF, Overman SB, Ribes JA. (1-3)- $\beta$-d-Glucan assay: a review of its laboratory and clinical application. Lab Med 2011;42(11):679-685. DOI: 10.1309/LM8BW8QNV7NZBROG. 